\title{
Effects of Testosterone, 17 $\beta$-Estradiol, and Downstream Estrogens on Cytokine Secretion from Human Leukocytes in the Presence and Absence of Cortisol
}

\author{
DAVID JANELE,${ }^{a}$ THOMAS LANG,${ }^{a}$ SILVIA CAPELLINO ${ }^{a}{ }^{\text {MAURIZIO }}$ \\ CUTOLO,${ }^{b}$ JOSE ANTONIO P. DA SILVA, ${ }^{c}$ AND RAINER H. STRAUB ${ }^{a}$
}

${ }^{a}$ Laboratory of Neuroendocrinoimmunology, Department of Internal Medicine I, University Hospital, 93042 Regensburg, Germany

${ }^{b}$ Division of Rheumatology, Department of Internal Medicine and Medical Specialities, University of Genova, Genova, Italy

${ }^{c}$ Department of Medicine III and Rheumatology, Coimbra University Hospital, Coimbra, Portugal

\begin{abstract}
Estrogens at physiological concentrations are thought to play an immune-stimulating role, whereas androgens have an antiinflammatory impact. However, their role on cytokine secretion in the presence or absence of cortisol has not been investigated. Furthermore, the role of hydroxylated estrogens downstream of 17 $\beta$-estradiol (E2) on secretion of tumor necrosis factor (TNF) is not known. In this study on peripheral blood leukocytes of healthy male subjects, we scrutinized the influence of prior sex hormones (for $24 \mathrm{~h}$ ) with and without later addition of cortisol (for another $24 \mathrm{~h}$ ) on stimulated secretion of TNF, IL-2, IL-4, IL-6, IL-10, and interferon- $\gamma$ (IFN- $\gamma)$. E2 stabilized or increased immune stimuli-induced secretion of TNF, IL-2, IL-4, IL-6, IL-10, and IFN $\gamma$ in relation to testosterone. Testosterone, in contrast, inhibited (IL2, IL-4, IL-10) or tended to inhibit stimulated secretion of these cytokines (TNF, IFN $\gamma$ ). This effect of E2 was pronounced at a concentration of $10^{-10} \mathrm{M}$ (testosterone: $\left.10^{-7} \mathrm{M}\right)$ in the presence of cortisol. E2 $\left(10^{-8} \mathrm{M}\right.$, $\left.10^{-10} \mathrm{M}\right)$ and testosterone $\left(10^{-7} \mathrm{M}\right)$ did not change glucocorticoid receptor expression. The downstream estrogens $2 \mathrm{OH}$-estradiol(one), $4 \mathrm{OH}$ estradiol(one), and $160 \mathrm{H}$-estradiol(one) did not stimulate TNF secretion at $10^{-10} \mathrm{M}$, but even inhibited its secretion at $10^{-11} \mathrm{M}$. However, the combination of $16 \mathrm{OH}$-estradiol(one) on one side and $2 \mathrm{OH}$-estradiol(one) or 4OH-estradiol(one) on the other side markedly stimulated TNF secretion that was only observable in the presence of cortisol. In conclusion, at physiological concentrations, $\mathrm{E} 2$ and a combination of downstream estrogens
\end{abstract}

Address for correspondence: Rainer H. Straub, M.D., Laboratory of Neuroendocrinoimmunology, Department of Internal Medicine I, University Hospital, 93042 Regensburg, Germany. Voice: +49941 944 7120; fax: +499419447121.

e-mail: rainer.straub@klinik.uni-regensburg.de

Ann. N.Y. Acad. Sci. 1069: 168-182 (2006). (C) 2006 New York Academy of Sciences. doi: 10.1196/annals.1351.015 
stabilized or increased immune stimuli-induced TNF secretion. These effects are dependent on the presence of physiological concentrations of cortisol. This study underlines the proinflammatory role of E2, which is probably dependent on conversion to a proinflammatory cocktail of downstream estrogens and the presence of cortisol.

KEYWORDS: testosterone; 17 $\beta$-estradiol; 2-hydroxyestrogens; 4-hydroxyestrogens; 16-hydroxyestrogens; cortisol; TNF; cytokines

\section{INTRODUCTION}

The pro- and anti-inflammatory effects of $17 \beta$-estradiol (E2) and other estrogens on secretion of proinflammatory cytokines have been a matter of debate for two decades. Authors in the field of rheumatology investigated estrogens in the context of a strong proinflammatory microenvironment and came to the conclusion that estrogens most often exert a proinflammatory influence on chronic inflammatory diseases, whereas androgens are considered to be anti-inflammatory. ${ }^{1,2}$ The proinflammatory influence of estrogens was regarded to be a major factor for the known female-to-male preponderance in autoimmune diseases. ${ }^{1,3-5}$ On the other hand, researchers in the field of bone research demonstrated inhibitory effects of estrogens on cytokine secretion in a noninflamed microenvironment leading to osteoprotective effects. ${ }^{6,7}$ This discrepancy probably depends on very different conversion of estrogens to downstream metabolites.

A proinflammatory microenvironment leads to conversion of androgens to E2 and downstream estrogens ${ }^{8}$ that are further converted to 16-hydroxylated active estrogens but not to 2-hydroxylated endogenous anti-estrogens. ${ }^{9,10}$ In contrast, in normal macrophages, conversion of androgens to estrogens is much less pronounced and 16-hydroxylated estrogens were not detected. ${ }^{11}$ Furthermore, in osteopenic postmenopausal women without inflammation, 16-hydroxylated estrone levels are much lower and correlated positively with bone mineral density, whereas levels of 2-hydroxylated anti-estrogens showed a negative correlation with bone mineral density. ${ }^{12,13}$ This indicates that the relation of 16-hydroxylated to 2-hydroxylated estrogens is completely different in chronic inflammatory diseases as compared to the situation in postmenopausal women with osteoporosis and without inflammation.

Breast cancer research revealed a mitogenic tumor growth-stimulating role of $16 \alpha$-hydroxylated estrogens, which indicates the potent estrogenic activity of these hormones. ${ }^{14}$ In vivo animal studies demonstrated a strong TNFincreasing role of 16-hydroxyestradiol (estriol). ${ }^{15,16}$ Other conversion products of estrone and $17 \beta$-estradiol are the 2-hydroxylated estrogens, such as 2-hydroxyestrone and 2-hydroxyestradiol. In contrast to 16 $\alpha$-hydroxylated estrogens, the 2-hydroxylated forms inhibit growth-promoting effects of $17 \beta$-estradiol. ${ }^{17}$ Furthermore, the estrogen metabolism pathway favoring 
2-hydroxylation over $16 \alpha$-hydroxylation is associated with a reduced risk of invasive breast cancer in premenopausal women. ${ }^{18}$ We can summarize that $16 \alpha$-hydroxylated estrogens are biologically active, proinflammatory, and proproliferative, whereas the 2-hydroxylated metabolites act as naturally occurring estrogen antagonists.

Apart from the very different metabolism of E2 to downstream estrogens under inflamed and noninflamed conditions, the presence of glucocorticoids may play an additional role for effects of sex hormones on proinflammatory cytokine secretion from peripheral blood leukocytes and local macrophages. At least on the level of the hypothalamus and pituitary gland, it has been demonstrated that females mount a stronger hypothalamic-pituitary-adrenal (HPA) axis response, and gonadectomy diminishes or abrogates this effect. ${ }^{2,19}$ It was discussed that feedback inhibition by cortisol of the hypothalamus or the pituitary gland may be modulated by sex hormones. ${ }^{2,19}$ The possibility that similar interactions between sex hormones and cortisol occur in peripheral leukocytes would have implications in understanding the known gender dimorphism of the immune and neuroendocrine system.

In this study, we focussed on the role of testosterone and E2 on cytokine secretion from human peripheral blood leukocytes in the presence and absence of cortisol. For this pilot study, we used blood leukocytes because of their availability in healthy subjects. Furthermore, we studied effects on cytokine secretion caused by downstream E2 metabolites: 16-hydroxylated estrogens, 2-hydroxylated estrogens, and 4-hydroxylated estrogens. In addition, we investigated whether or not E2 and testosterone modulate glucocorticoid receptor expression.

\section{MATERIALS AND METHODS}

\section{Subjects and Blood Samples}

For cytokine secretion studies, 9 (age 24-40 years) and, for analysis of glucocorticoid receptor expression, 15 (24-40 aged) healthy male subjects were recruited. All subjects were informed about the purpose of the experiments and gave informed consent for further use of blood samples. Blood was drawn in heparinized tubes between 9:00 and 10:00 AM and immediately processed.

\section{Culture Conditions and Application of Sex Hormones and Cortisol}

For cytokine secretion studies, a defined volume of whole blood was cultured in 48-well plates together with serum-free medium (whole blood assay in RPMI 1640 without phenol red, $0.57 \mathrm{mM}$ ascorbic acid, Sigma Aldrich, Taufkirchen, Germany). Preliminary assays demonstrated optimal cytokine secretion when using $100 \mu \mathrm{L}$ of whole blood (for IL-2, IL-4, IL-10, IFN $\gamma$, and 
TNF) and $25 \mu \mathrm{L}$ of whole blood (for IL-6) in an end volume of 1,250 $\mu \mathrm{L}$ (TABLE 1). During the first $24 \mathrm{~h}$, cells were incubated with vehicle, testosterone $\left(10^{-7}\right.$ and $10^{-8} \mathrm{~mol} / \mathrm{L}$, Sigma Aldrich), E2 $\left(10^{-8}\right.$ and $10^{-10} \mathrm{~mol} / \mathrm{L}$, Sigma Aldrich), 2-hydroxyestradiol $\left(10^{-10}\right.$ and $10^{-11} \mathrm{~mol} / \mathrm{L}$, Steraloids, Newport, Rhode Island, USA), 2-hydroxyestrone $\left(10^{-10}\right.$ and $10^{-11} \mathrm{~mol} / \mathrm{L}$, Steraloids $)$, 4-hydroxyestradiol $\left(10^{-10}\right.$ and $10^{-11} \mathrm{~mol} / \mathrm{L}$, Steraloids), 4-hydroxyestrone $\left(10^{-10}\right.$ and $10^{-11} \mathrm{~mol} / \mathrm{L}$, Steraloids), 16-hydroxyestradiol $\left(10^{-10}\right.$ and $10^{-11}$ $\mathrm{mol} / \mathrm{L}$, Steraloids), and 16-hydroxyestrone $\left(10^{-10}\right.$ and $10^{-11} \mathrm{~mol} / \mathrm{L}$, Steraloids). After $24 \mathrm{~h}$, the culture medium was removed and substituted with new medium without sex hormones, carrying lipopolysaccharide (LPS for IL-6 stimulation, $0.5 \mathrm{ng} / \mathrm{mL}$, Sigma Aldrich) or concanavalin A $(100 \mu \mathrm{g} / \mathrm{mL}$, for stimulation of other cytokines, Sigma Aldrich), two strong immune stimuli, with or without cortisol (hydrocortisone, $10^{-6} \mathrm{~mol} / \mathrm{L}$, Pharmacia Upjohn, Karlsruhe, Germany). We used cortisol at $10^{-6} \mathrm{~mol} / \mathrm{L}$ because in preceding whole blood assays with naturally available binding proteins this concentration yielded half-maximum inhibition of TNF secretion (without cortisol: $109.1 \pm$ $9.7 \mathrm{pg} \mathrm{TNF} / \mathrm{mL}$ [100\%] vs. with cortisol: $79.4 \pm 5.1 \mathrm{pg} \mathrm{TNF} / \mathrm{mL}[73 \%]$ ). These first assays also demonstrated that the observed decrease of about $27 \%$ reflects the half-maximum effect of cortisol. After 12 and $24 \mathrm{~h}$, supernatants were taken and stored at $-30^{\circ} \mathrm{C}$. TABLE 1 summarizes applied conditions.

For analysis of the influence of sex hormones on glucocorticoid receptor expression, $30 \mathrm{~mL}$ of heparinized whole blood were cultured in $25 \mathrm{~cm}^{2}$ culture flasks $(70 \mathrm{~mL})$ for $24 \mathrm{~h}$ with vehicle, testosterone $\left(10^{-7} \mathrm{~mol} / \mathrm{L}\right)$, or $17 \beta$ estradiol $\left(10^{-8}\right.$ and $10^{-10} \mathrm{~mol} / \mathrm{L}$, Sigma Aldrich). In these experiments, no cortisol was added. After $24 \mathrm{~h}$, cultured cells were re-suspended, centrifuged, treated twice with distilled water to remove erythrocytes, and washed twice in phosphate-buffered saline (Sigma Aldrich). Then, leukocytes were lysed for 30 min in lysis buffer containing PMSF, SDS, NP-40, sodium deoxycholate, and a mixture of protein inhibitors ("complete" from Roche Diagnostics, Mannheim, Germany). After centrifuging, supernatants were stored at $-30^{\circ} \mathrm{C}$. Protein concentrations were measured using the BCA assay (reagents from Sigma Aldrich).

TABLE 1. Mode of stimulation, use of whole blood, and observation time for different cytokines

\begin{tabular}{lccc}
\hline Cytokine & $\begin{array}{c}\text { Volume of } \\
\text { WB }[\mu \mathrm{L}]\end{array}$ & Stimulation & $\begin{array}{c}\text { Observation } \\
\text { Time }[\mathrm{h}]\end{array}$ \\
\hline IL-2 & 100 & ConA $100 \mu \mathrm{g} / \mathrm{mL}$ & 24 \\
IL-4 & 100 & ConA $100 \mu \mathrm{g} / \mathrm{mL}$ & 24 \\
IL-6 & 25 & LPS $0.5 \mathrm{ng} / \mathrm{mL}$ & 12 \\
IL-10 & 100 & ConA $100 \mu \mathrm{g} / \mathrm{mL}$ & 12 \\
IFN $\gamma$ & 100 & ConA $100 \mu \mathrm{g} / \mathrm{mL}$ & 24 \\
TNF $\alpha$ & 100 & ConA $100 \mu \mathrm{g} / \mathrm{mL}$ & 12 \\
\hline
\end{tabular}




\section{Cytokine Determination in Supernatants}

The cytokines IL-2, IL-4, IL-6, IL-10, IFN $\gamma$, and TNF were measured by immunometric enzyme immunoassays making use of antibody pairs (OptEIA ${ }^{\mathrm{TM}}$, BD Pharmingen, San Diego, CA). In our hands, intra- and inter-assay coefficients of variation were below $10 \%$. The detection limit was approximately 4-20 pg/mL depending on the cytokine tested.

\section{Western Blotting}

Gels (Novex 8\% Tris-Glycine, Invitrogen, Karlsruhe, Germany) were loaded with $25 \mu \mathrm{g}$ of protein and, after electrophoretic processing, transferred to nitro-cellulose. Membranes were blocked in Tris-buffered saline (TBS) containing $2.5 \%$ of human serum protein and $2.5 \%$ of bovine serum albumin (Biomol, Hamburg, Germany). Blots were then incubated for $1 \mathrm{~h}$ at room temperature with primary antibodies against the human glucocorticoid receptor (1:500 in 5\% nonfat dry milk in TBS, Affinity Bioreagents, Golden, Colorado, USA). Subsequently, membranes were washed in TBS and incubated with secondary antibodies (1:1000 in TBS, horseradish peroxidase-conjugated, Affinity Bioreagents). After another washing step, blots were developed using chemiluminescent reagents (ECL+, Amersham Biosciences, Amersham, UK) and then subjected to autoradiography. The films (Hyperfilm, Amersham, UK) were scanned and analyzed using a densitometric method (Molecular Dynamics, Amersham, UK).

\section{Statistical Analysis and Data Presentation}

Data are given as the mean \pm SEM. Medians were compared by MannWhitney test (SPSS/PC for Windows 11.0, SPSS, Chicago, IL).

\section{RESULTS}

\section{Influence of Testosterone and E2 on Cytokine Secretion in the Presence and Absence of Cortisol}

In order to test the influence of testosterone and E2 on cytokine secretion, we used physiological concentrations of these hormones in a relatively natural microenvironment (whole blood). In the presence of cortisol, E2, in relation to testosterone, at concentrations of $10^{-8}$ and $10^{-10} \mathrm{~mol} / \mathrm{L}$ stabilized secretion of TNF, IL-2, IFN $\gamma$, and IL-10 (FIGs. 1A, 1C, 1D, and 2B). For IL-6 and IL-4, only E2 at $10^{-10} \mathrm{~mol} / \mathrm{L}$ exerts similar effects (Figs. $1 \mathrm{~B}$ and $2 \mathrm{~A}$ ). E2 at $10^{-10}$ 
(A)

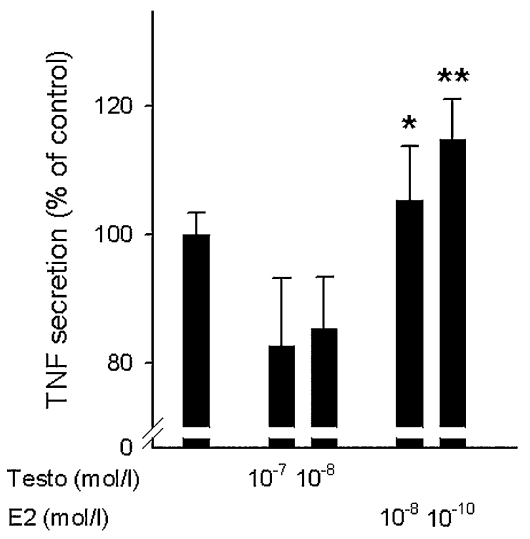

(C)

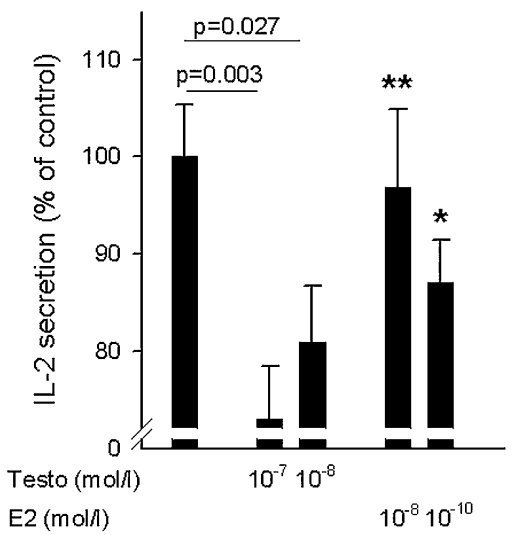

(B)

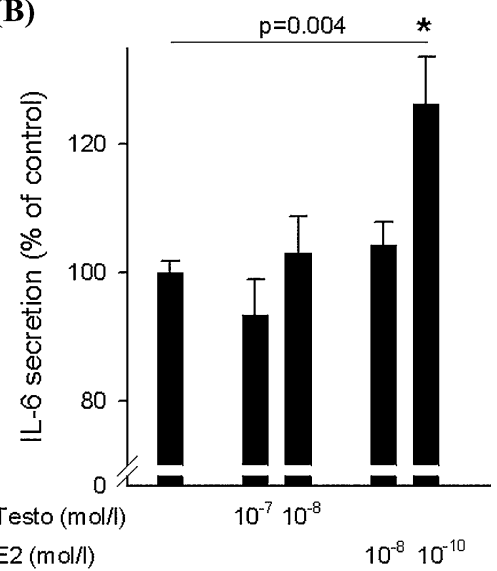

(D)

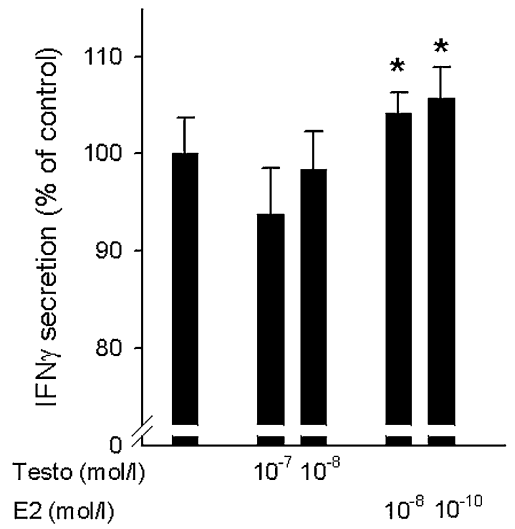

FIGURE 1. Modulation of cytokine secretion by $17 \beta$-estradiol (E2) and testosterone in the presence of cortisol. Whole blood of five healthy male subjects was incubated in triplicate with vehicle, testosterone, and E2 for $24 \mathrm{~h}$ at indicated concentrations. After $24 \mathrm{~h}$, medium was replaced and cells were stimulated with $0.5 \mathrm{ng} / \mathrm{mL}$ LPS (IL-6) or $100 \mu \mathrm{g} / \mathrm{mL}$ concanavalin A (TNF, IL-2, IFN $\gamma$ ) for further $12 \mathrm{~h}$ (IL-6, TNF) or $24 \mathrm{~h}$ (IL-2, IFN $\gamma$ ) in the presence of $10^{-6} \mathrm{~mol} / \mathrm{L}$ cortisol. Supernatants were removed and cytokines were measured by enzyme-linked immunosorbent assay (ELISA). Means \pm SEM are shown for TNF (A: control $=79.4 \pm 5.1 \mathrm{pg} / \mathrm{mL})$, IL-6 $(\mathbf{B}$ : control $=857 \pm 137 \mathrm{pg} / \mathrm{mL})$, IL-2 $(\mathbf{C}$ : control $=$ $289 \pm 27 \mathrm{pg} / \mathrm{mL}$ ), and IFN $\gamma(\mathbf{D}$ : control $=521 \pm 49 \mathrm{pg} / \mathrm{mL}) .{ }^{*} P<0.05,{ }^{* *} P<0.01$ versus the respective concentration of testosterone.

$\mathrm{mol} / \mathrm{L}$ stimulated IL-6 secretion in relation to vehicle (FIG. 1B). With respect to other cytokines, E2 had no stimulating activity in relation to vehicle (FIGS. 1 and 2). In the absence of $10^{-6} \mathrm{~mol} / \mathrm{L}$ cortisol, we observed very similar effects for IL-2, IL-4, IL-6, and IL-10 (data not shown). 
(A)

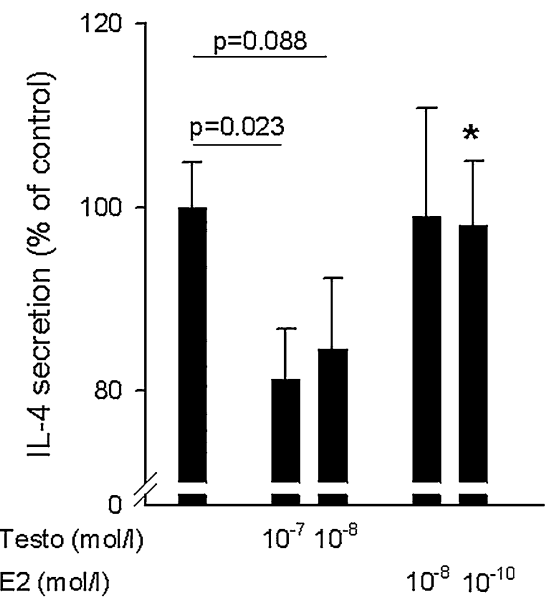

(B)

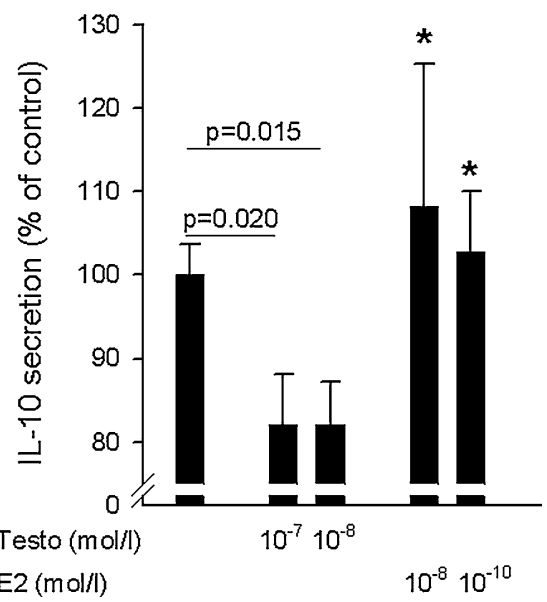

FIGURE 2. Modulation of cytokine secretion by $17 \beta$-estradiol (E2) and testosterone in the presence of cortisol. Means \pm SEM are shown for IL-4 (A: control $=94.5 \pm 13.5$ $\mathrm{pg} / \mathrm{mL})$ and IL-10 (B: control $=127.1 \pm 8.4 \mathrm{pg} / \mathrm{mL})$. Whole blood of five healthy male subjects was incubated in triplicate with vehicle, testosterone, and E2 for $24 \mathrm{~h}$ at indicated concentrations. After $24 \mathrm{~h}$, medium was replaced and cells were stimulated with $100 \mu \mathrm{g} / \mathrm{mL}$ concanavalin A for further $24 \mathrm{~h}$ in the presence of $10^{-6} \mathrm{~mol} / \mathrm{L}$ cortisol. ${ }^{*} P<0.05$ versus respective concentration of testosterone.

With respect to testosterone, in the presence of cortisol, this hormone at $10^{-7}$ and $10^{-8} \mathrm{~mol} / \mathrm{L}$ inhibited secretion of IL-2, IL-4, and IL-10 (FIGS. 1C, $2 \mathrm{~A}$, and 2B), and it tended to inhibit TNF and IFN $\gamma$ (FIGs. 1A and 1D) but not IL-6 (FIG. 1B). Similar effects on IL-2, IL-4, and IL-10 were seen in the absence of cortisol (data not shown).

\section{Influence of Testosterone and E2 on Glucocorticoid Receptor Expression}

Neither testosterone at $10^{-7} \mathrm{~mol} / \mathrm{L}$ nor E2 at $10^{-8}$ and $10^{-10} \mathrm{~mol} / \mathrm{L}$ changed glucocorticoid receptor expression (FIG. 3).

\section{Effects of Downstream Metabolites of E2 on TNF Secretion}

Since metabolites of estrogens may modulate cytokine secretion, we investigated effects of various metabolites of E2 and their combinations on TNF secretion in the presence of cortisol. Metabolites were used in concentrations similar to those E2 (or lower) because their maximum concentration is expected to be equal to or lower than the concentration of the precursor E2. In the presence of 


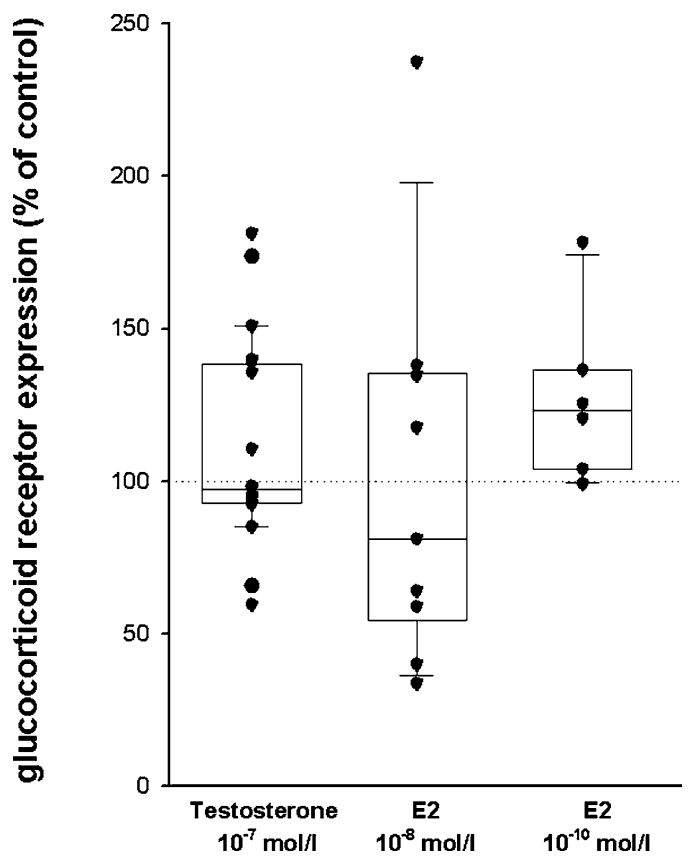

FIGURE 3. Influence of testosterone and $17 \beta$-estradiol (E2) on glucocorticoid receptor expression in peripheral blood leukocytes. The values are given in percent densitometric control, which is indicated by dotted horizontal line. The boundary of the box closest to zero indicates the 25 th percentile, a line within the box marks the median, and the boundary of the box farthest from zero indicates the 75 th percentile. Whiskers above and below the box indicate the 90th and 10th percentiles. Black symbols give the individual values of participating subjects (for T at $10^{-7} M=9$, for E2 at $10^{-8} M=9$, and for E2 at $10^{-10} M$ $=6)$.

cortisol, all investigated metabolites at $10^{-10} \mathrm{~mol} / \mathrm{L}$ did not change TNF secretion (FIG. 4A). Thus, these effects were similar as compared to E2 (compare Fig. 1A). Interestingly, sole application of 2-hydroxyestradiol, 2-hydroxyestrone, 4-hydroxyestrone, or 16-hydroxyestradiol at $10^{-11} \mathrm{~mol} / \mathrm{L}$ inhibited secretion of TNF (FIg. 4A). Similarly, sole application of 4-hydroxyestradiol or 16hydroxyestrone tended to inhibit TNF secretion (FIG. 4A).

One molecule of E2 may be converted to different downstream metabolites. In order to further delineate effects of combinations of estrogens downstream of E2, 16-hydroxyestradiol and 16-hydroxyestrone at $10^{-10} \mathrm{~mol} / \mathrm{L}$ were combined with the other metabolites. It is obvious that combinations of 16hydroxyestradiol (16OHE2) at $10^{-10} \mathrm{~mol} / \mathrm{L}$ and 2- and 4-hydroxylated estrogens at $10^{-11} \mathrm{~mol} / \mathrm{L}$ markedly increased TNF secretion (FIG. 4B). Thus, a preponderance of 16-hydoxyestradiol over 2-/4-hydroxylated estrogens using a ratio of 10:1 increased TNF secretion. A similar increase was observed for the combination of 16-hydroxyestrone (16OHE1) and 4-hydroxylated 


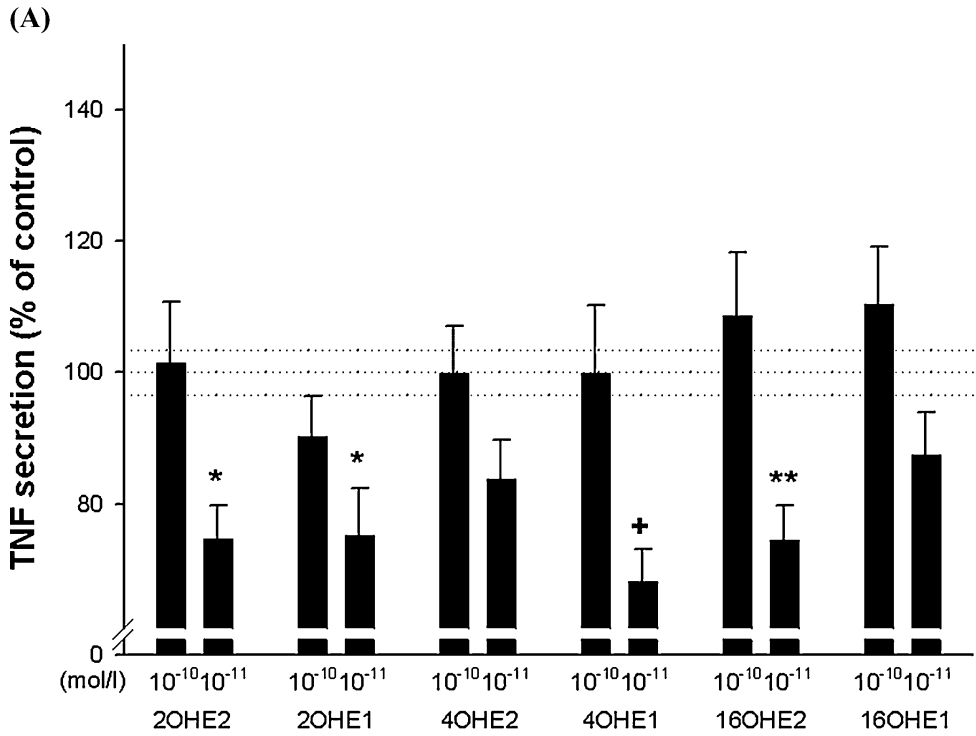

(B)

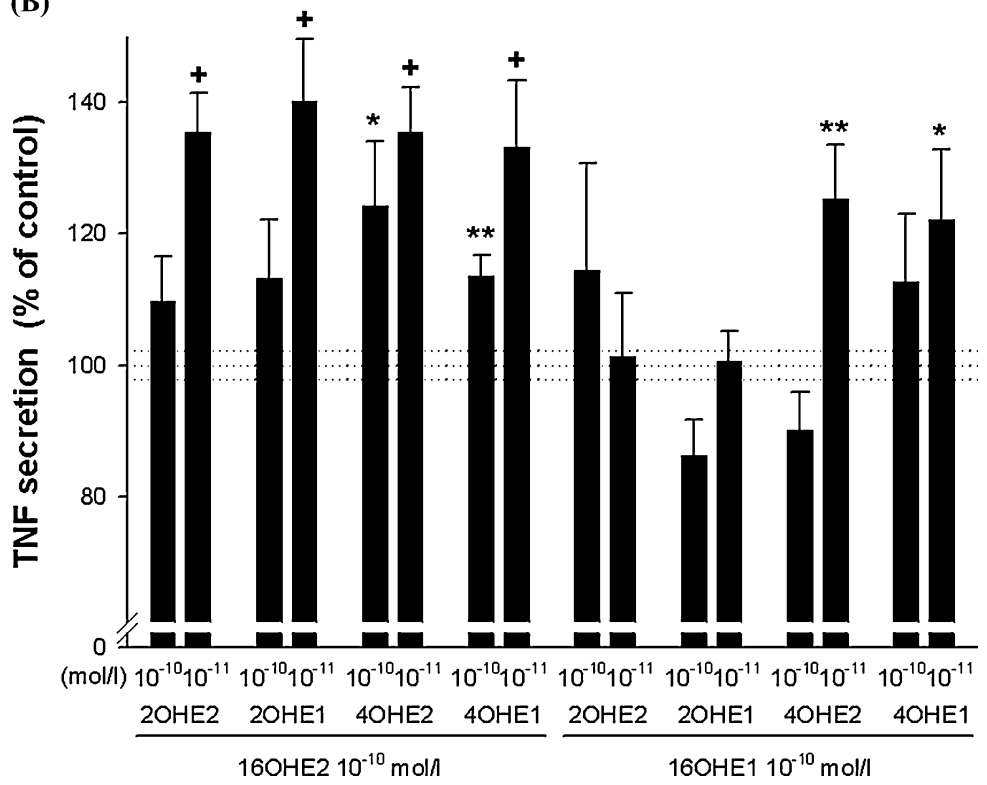

FIGURE 4. Influence of downstream metabolites of $17 \beta$-estradiol (E2) on secretion of TNF. (A) Influence of individual metabolites on TNF secretion. Control TNF was 54.1 \pm $5.2 \mathrm{pg} / \mathrm{mL}$. (B) Influence of combinations of metabolites on TNF secretion. Control TNF was $21.8 \pm 2.1 \mathrm{pg} / \mathrm{mL}$. For both panels: Data are given as means $\pm \mathrm{SEM}$ in percent of control. ${ }^{*} P<0.05,{ }^{* *} P<0.01,+P<0.001$ versus control. Abbreviations: 2OHE2, 2-hydroxyestradiol; 2OHE1, 2-hydroxyestrone; 4OHE2, 4-hydroxyestradiol; 4OHE1, 4hydroxyestrone; 16OHE2, 16-hydroxyestradiol; 16OHE1 (= estriol), 16-hydroxyestrone. 
TABLE 2. Effect of the combination of 16-hydroxylated estrogens and 2-/4-hydroxylated estrogens on secretion of TNF in the absence of cortisol.

\begin{tabular}{llc}
\hline $\begin{array}{l}\text { 16-Hydroxylated } \\
\text { Estrogen }\end{array}$ & \multicolumn{1}{c}{$\begin{array}{c}\text { 2-/4-Hydroxylated } \\
\text { Estrogen }\end{array}$} & $\begin{array}{c}\text { TNF Secretion } \\
\text { (\% of Control) }\end{array}$ \\
\hline- & & Control: $100 \pm 4.2$ \\
$16 \mathrm{OH}$-estradiol $10^{-10} \mathrm{~mol} / \mathrm{L}$ & 2OH-estradiol $10^{-11} \mathrm{~mol} / \mathrm{L}$ & $91.4 \pm 10.9$ \\
& 2OH-estrone $10^{-11} \mathrm{~mol} / \mathrm{L}$ & $77.7 \pm 10.1$ \\
& 4OH-estradiol $10^{-11} \mathrm{~mol} / \mathrm{L}$ & $100.8 \pm 7.6$ \\
& $4 \mathrm{OH}$-estrone $10^{-11} \mathrm{~mol} / \mathrm{L}$ & $123.2 \pm 16.5$ \\
$16 \mathrm{OH}$-estrone $10^{-10} \mathrm{~mol} / \mathrm{L}$ & 2OH-estradiol $10^{-11} \mathrm{~mol} / \mathrm{L}$ & $66.8 \pm 5.7^{*}$ \\
& 2OH-estrone $10^{-11} \mathrm{~mol} / \mathrm{L}$ & $54.5 \pm 6.8^{* *}$ \\
& $4 \mathrm{OH}$-estradiol $10^{-11} \mathrm{~mol} / \mathrm{L}$ & $104.1 \pm 5.2$ \\
& $4 \mathrm{OH}$-estrone $10^{-11} \mathrm{~mol} / \mathrm{L}$ & $119.5 \pm 24.7$ \\
\hline
\end{tabular}

Data are given as means \pm SEM.

Control TNF $=55.0 \pm 4.5 \mathrm{pg} / \mathrm{mL} .{ }^{*} P<0.01,{ }^{* *} P<0.001$ for the comparison versus control.

metabolites but not for 2-hydroxylated estrogens (FIG. 4B). Such a stimulatory effect of combinations of downstream metabolites was not observed in the absence of cortisol (TABLE 2). In contrast, in the absence of cortisol, the combination of 16-hydroxyestrone and 2-hydroxylated estrogens even inhibited TNF secretion (TABLE 2).

\section{DISCUSSION}

This study showed dichotomous effects of sex hormones: E2 and metabolites stabilized or increased cytokine secretion whereas testosterone inhibited this secretion. This dual role of estrogens and testosterone on cytokine secretion has been described in the literature and it was thought that this phenomenon could explain the well-known female-to-male preponderance in acquiring autoimmune diseases, particularly when TNF and IFN $\gamma$ play a dominant diseaseperpetuating role. ${ }^{1,3-5}$ However, two aspects have not been studied so far.

First, the additional role of cortisol for sex hormone modulation of cytokines from peripheral blood leukocytes has been unclear. This can be important because these mechanisms may explain interactions of hormones on the level not only of the pituitary gland, but also in peripheral inflammation. We demonstrated that a stimulus of the HPA axis (injection of IL- $1 \beta$, ether anesthesia, or granulomatous disease) led to stronger corticosterone responses in female than in male mice, ${ }^{19}$ which corroborated a similar study in humans. ${ }^{20}$ Evidence from the literature strongly supports the concept that estrogens enhance and androgens diminish the glucocorticoid response to a variety of inflammatory and noninflammatory stimuli. Although the mechanisms for these interactions are not fully elucidated, there is strong evidence that sex steroids affect the expression of glucocorticoid receptors in the central nervous system and 
modulate the negative feedback exerted by cortisol at the hypothalamus. ${ }^{2,19}$ These observations led us to hypothesize that cortisol interacts with testosterone or estradiol on the cellular level, which can be studied in humans using peripheral blood leukocytes. This study demonstrates that E2 (in relation to testosterone) stimulated production of TNF, IL-6, IL-2, IFN $\gamma$, IL-4, and IL-10 caused by the proinflammatory influence of applied immune stimuli (LPS and Con A). The question arises as to how these findings may be linked to the estrogenic support of the HPA axis response.

It has been demonstrated that the pituitary folliculo-stellate cell is a macrophage type of cell located in the pituitary gland. ${ }^{21,22}$ This type of cell, similar to peripheral blood monocytes/macrophages, can be stimulated by immune stimuli in order to produce proinflammatory cytokines locally. ${ }^{23,24}$ Locally produced cytokines can act as secretagogues for ACTH and other pituitary hormones. ${ }^{24}$ In this respect, the folliculo-stellate cell may be an important local linking element between immune stimuli and hormone production. ${ }^{24}$ In this present study, we have shown that E2 stabilized (in relation to testosterone) or increased cytokine secretion induced by stimulatory agents in peripheral blood cells. A similar effect upon the folliculo-stellate cell might explain the facilitating effects of E2 on pituitary hormone secretion and the inhibitory effect of testosterone via an increase or decrease of locally produced cytokines, respectively.

The second open question that was addressed in this study is the role of downstream metabolites of E2 on stimulated TNF secretion. Our results demonstrate that the ratio of 16-hydroxylated estrogens in relation to 2-/4-hydroxylated estrogens is important for TNF secretion. We were able to describe that a ratio of 10:1 of 16-hydroxyestradiol in relation to 2-/4-hydroxylated estrogens markedly stimulated TNF secretion in the presence of cortisol. This effect was not observed in the absence of cortisol. Furthermore, in the absence of cortisol, the combination of 16-hydroxyestrone and 2-hydroxylated estrogens even strongly inhibited TNF secretion. These results delineate the importance of downstream estrogens in respective concentrations. Again, this may play a role not only on the pituitary level, but also in the peripheral immune response. As pointed out in the introduction to this article, in persons with chronic inflammatory diseases, we observe a large shift toward 16-hydroxylated estrogens in comparison to that measure in postmenopausal osteopenic women. ${ }^{9}{ }^{9}, 10,12,13 \mathrm{Au}-$ thors in the field of rheumatology trust that estrogens exert proinflammatory effects in most rheumatic diseases, whereas authors in bone research believe that estrogens prevent osteoporosis through inhibition of cytokine secretion. We suggest the following model to find an answer for this obvious discrepancy (FIG. 5):

In the noninflammatory situation, an osteopenia-inducing milieu (FIG. 5, left panel) is characterized by increased levels of 2-hydroxylated estrogens and low levels of 16-hydroxylated estrogens. ${ }^{12,13}$ This balance would diminish the basal secretion of TNF, IL-1, and IL-6 and, therefore, avoid bone loss 


\section{non-inflamed}

\section{chronically inflamed}

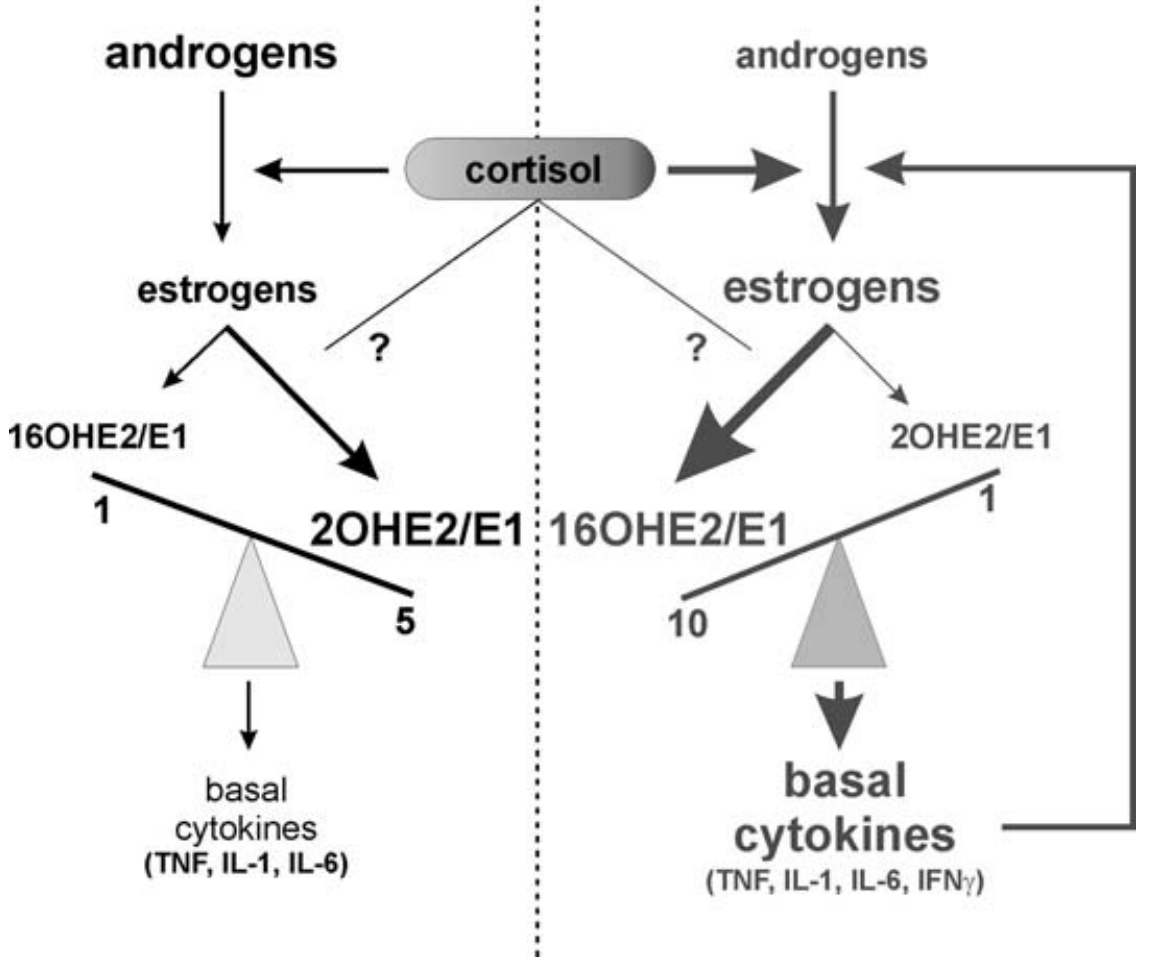

FIGURE 5. Model of the estrogen influence on secretion of proinflammatory cytokines in a noninflamed and chronically inflamed microenvironment. For abbreviation of estrogens see legend to FIGURE 4. Left panel: In the noninflamed situation, an osteopenia-inducing milieu is characterized by increased levels of 2OHE1/E2 and low levels of 16OHE1/E2. In this situation, basal secretion of TNF, IL-1, and IL-6 is osteoclastogenic. However, TNF, IL-1 and IL-6 are markedly lower than in chronically inflamed tissue. In such a situation, administration of therapeutic estrogens probably increases the 2OHE1/E2 because this particular pathway is switched on. This would be a negative signal for the osteoclastogenic TNF secretion. Right panel: In contrast, during chronic inflammation in the situation of rheumatic diseases, such as rheumatoid arthritis, the balance is switched to 16OHE1/E2. Under these conditions, one can assume that therapeutic administration of estrogens would enhance 16OHE1/E2 over 2OHE1/E2, which would support secretion of proinflammatory cytokines. Cortisol itself is a stimulator of aromatase, and somewhat elevated cortisol concentrations in the tissue, although inadequately low in relation to inflammation, would promote estrogen-induced secretion of proinflammatory TNF. Thus, under inflamed conditions estrogens appear as proinflammatory agents whereas under noninflamed conditions estrogens appear as anti-inflammatory. 
(for review see Ref. 6). In chronic inflammation (FIG. 5, right panel), administration of therapeutic estrogens probably increases the 16-hydroxylated estrogens because this particular pathway is switched on. During estrogen therapy of postmenopausal women without inflammation, the 2-hydroxylated estrogens would appear in higher concentrations compared to 16-hydroxylated estrogens, which would be a negative signal for the osteoclastogenic TNF secretion (FIG. 5, left panel). In contrast, during chronic inflammation in the situation of rheumatic diseases, such as rheumatoid arthritis, the balance is switched to estrogens and particularly to 16-hydroxylated estrogens (FIG. 5, right panel). As shown in this study, a preponderance of 16-hydroxyestradiol versus 2-/4hydroxylated estrogens in the presence of cortisol markedly increased TNF secretion. In this situation, one can assume that therapeutic administration of estrogens would enhance 16-hydroxylated estrogens over 2-/4-hydroxylated estrogens, which would support secretion of proinflammatory cytokines (FIG. 5 , right panel). Cortisol itself promotes the conversion of androgens to estrogens. $^{25}$ Therefore, somewhat elevated cortisol concentrations in the tissue ${ }^{26}$ would result in higher estrogen levels and, indirectly, increased secretion of proinflammatory TNF. Thus, under inflammatory conditions estrogens appear as proinflammatory agents.

In conclusion, this study on peripheral blood leukocytes demonstrated that E2 stabilized or increased and testosterone inhibited immune stimuli-induced secretion of proinflammatory cytokines, such as TNF and IFN $\gamma$. Furthermore, combined application of 16-hydroxylated estrogens and 2-/4-hydroxylated estrogens in a ratio of 10:1 increased TNF secretion only in the presence of cortisol. The findings of this study shed new light on the gender dimorphism of the neuroendocrine and immune systems. Furthermore, it demonstrated that distinct cocktails of downstream E2 metabolites may induce pro- or anti-inflammatory responses in blood leukocytes. Further studies in folliculostellate cells and synovial cells of patients with rheumatoid arthritis are needed in order to further elucidate the importance of these findings on the local level.

\section{ACKNOWLEDGMENT}

This study was supported by a grant from the Deutsche Forschungsgemeinschaft (Str 511/10 - 1).

\section{REFERENCES}

1. Cutolo, M. \& R. Wilder. 2000. Different roles for androgens and estrogens in the susceptibility to autoimmune rheumatic diseases. Rheum. Dis. Clin. North Am. 26: 825-839.

2. DA SILVA, J.A. 1995. Sex hormones, glucocorticoids and autoimmunity: facts and hypotheses. Ann. Rheum. Dis. 54: 6-16. 
3. TALAL, N. 1981. Sex steroid hormones and systemic lupus erythematosus. Arthritis Rheum. 24: 1054-1056.

4. KANDA, N. et al. 1999. Estrogen enhancement of anti-double-stranded DNA antibody and immunoglobulin $\mathrm{G}$ production in peripheral blood mononuclear cells from patients with systemic lupus erythematosus. Arthritis Rheum. 42: 328-337.

5. Straub, R.H. \& M. Cutolo. 2001. Involvement of the hypothalamic-pituitaryadrenal/gonadal axis and the peripheral nervous system in rheumatoid arthritis: viewpoint based on a systemic pathogenetic role. Arthritis Rheum. 44: 493-507.

6. Manolagas, S.C. \& R.L. JiLKA. 1995. Bone marrow, cytokines, and bone remodeling: emerging insights into the pathophysiology of osteoporosis. N. Engl. J. Med. 332: 305-311.

7. PACIFICI, R. 1996. Estrogen, cytokines, and pathogenesis of postmenopausal osteoporosis. J. Bone Miner. Res. 11: 1043-1051.

8. CastagnetTA, L.A. et al. 2003. Increased estrogen formation and estrogen to androgen ratio in the synovial fluid of patients with rheumatoid arthritis. J. Rheumatol. 30: 2597-2605.

9. LAHitA, R.G. et al. 1979. Alterations of estrogen metabolism in systemic lupus erythematosus. Arthritis Rheum. 22: 1195-1198.

10. WeIDLER, C. et al. 2004. Patients with rheumatoid arthritis and systemic lupus erythematosus have increased renal excretion of mitogenic estrogens in relation to endogenous antiestrogens. J. Rheumatol. 31: 489-494.

11. Schmidt, M. et al. 2000. Conversion of dehydroepiandrosterone to downstream steroid hormones in macrophages. J. Endocrinol. 164: 161-169.

12. LIM, S.K. et al. 1997. Altered hydroxylation of estrogen in patients with postmenopausal osteopenia. J. Clin. Endocrinol. Metab. 82: 1001-1006.

13. Leelawattana, R. et al. 2000. The oxidative metabolism of estradiol conditions postmenopausal bone density and bone loss. J. Bone Miner. Res. 15: 2513-2520.

14. SCHNEIDER, J. et al. 1984. Antiestrogen action of 2-hydroxyestrone on MCF-7 human breast cancer cells. J. Biol. Chem. 259: 4840-4845.

15. ZUCKERMAN, S.H. et al. 1996. Estriol: a potent regulator of TNF and IL-6 expression in a murine model of endotoxemia. Inflammation 20: 581-597.

16. IKEJIMA, K. et al. 1998. Estrogen increases sensitivity of hepatic Kupffer cells to endotoxin. Am. J. Physiol. 274: G669-G676.

17. VAndewalle, B. \& J. LeFeBVRE. 1989. Opposite effects of estrogen and catecholestrogen on hormone-sensitive breast cancer cell growth and differentiation. Mol. Cell Endocrinol. 61: 239-246.

18. Muti, P. et al. 2000. Estrogen metabolism and risk of breast cancer: a prospective study of the 2:16alpha-hydroxyestrone ratio in premenopausal and postmenopausal women. Epidemiology 11: 635-640.

19. DA SILVA, J.A. et al. 1993. Sex steroids affect glucocorticoid response to chronic inflammation and to interleukin-1. J. Endocrinol. 136: 389-397.

20. Kirschbaum, C. et al. 1996. Short-term estradiol treatment enhances pituitaryadrenal axis and sympathetic responses to psychosocial stress in healthy young men. J. Clin. Endocrinol. Metab. 81: 3639-3643.

21. Allaerts, W. et al. 1997. A population of interstitial cells in the anterior pituitary with a hematopoietic origin and a rapid turnover: a relationship with folliculostellate cells? J. Neuroimmunol. 78: 184-197.

22. Giometto, B. et al. 1997. Folliculo-stellate cells of human pituitary adenomas: immunohistochemical study of the monocyte/macrophage phenotype expression. Neuroendocrinology 65: 47-52. 
23. VANKelecom, H. et al. 1989. Production of interleukin-6 by folliculo-stellate cells of the anterior pituitary gland in a histiotypic cell aggregate culture system. Neuroendocrinology. 49: 102-106.

24. RENNER, U. et al. 1998. Regulation and role of intrapituitary IL-6 production by folliculostellate cells. Domest. Anim. Endocrinol. 15: 353-362.

25. Svenstrup, B. et al. 1990. Comparison of the effect of cortisol on aromatase activity and androgen metabolism in two human fibroblast cell lines derived from the same individual. J. Steroid Biochem. 35: 679-687.

26. StRAub, R.H. et al. 2002. Inadequately low serum levels of steroid hormones in relation to IL-6 and TNF in untreated patients with early rheumatoid arthritis and reactive arthritis. Arthritis Rheum. 46: 654-662. 